\title{
Factors predicting patient use of the emergency department: a retrospective cohort study
}

\author{
Jane McCusker MD DrPH, Pierre Tousignant MD MSc, Roxane Borgès Da Silva PhD, Antonio Ciampi PhD, \\ Jean-Frédéric Lévesque MD PhD, Alain Vadeboncoeur MD, Steven Sanche MSc
}

\begin{abstract}
Background: Many studies have shown the tendency for people without a regular care provider or primary physician to make greater use of emergency departments. We sought to determine the effects of three aspects of care provided by primary physicians (physician specialty, continuity of care and comprehensiveness of care) on their patients' use of the emergency department.

Methods: Using provincial administrative databases, we created a cohort of 367315 adults aged 18 years and older. Participants were residents of urban areas of Quebec. Affiliation with a primary physician, the specialty of this physician (i.e., family physician v. specialist), continuity of care (as measured using the Usual Provider Continuity index) and comprehensiveness of care (i.e., number of complete annual examinations) were measured among participants $(n=311701)$ who had visited a physician three or more times during a two-year baseline period. We used multivariable negative binomial regression to investigate the relationships between measures of care and the number of visits to emergency departments during a 12-month follow-up period.
\end{abstract}

Results: Among participants under 65 years of age, emergency department use was higher for those not affiliated than for those affiliated with a family physician (incidence rate ratio [IRR] 1.11, 95\% confidence interval [CI] 1.051.16) or a specialist (IRR $1.10,95 \% \mathrm{Cl} 1.04-$ 1.17). Among patients aged 65 years and older, having a specialist primary physician, as opposed to a family physician, predicted increased use of the emergency department (IRR 1.13, 95\% Cl 1.09-1.17). Greater continuity of care with a family physician predicted less use of the emergency department only among participants who made 25 or more visits to a physician during the baseline period. Greater continuity of care with a specialist predicted less use of the emergency department overall, particularly among participants with intermediate numbers of multimorbidities and admissions to hospital. Greater comprehensiveness of care by family physicians predicted less use of the emergency department.

Interpretation: Efforts to increase the proportion of adults affiliated with a family physician should target older adults, people who visit physicians more frequently and people with multiple comorbidities and admissions to hospital.
$\mathrm{R}$ eforming primary care in Canada has been stimulated in part by increased crowding of emergency departments and evidence that their use, particularly for nonurgent care, may be related to inadequate primary care in the community. ${ }^{1,2}$ Restructuring efforts, such as encouraging family physicians to work in multidisciplinary group practices with 24-hour access, are challenged by a relative shortage of family physicians. ${ }^{3}$ These issues are of particular importance in Quebec; despite relatively high numbers of family physicians per capita, in comparison with other provinces, residents of Quebec have the lowest rates of affiliation with a family physician and have one of the highest rates of seeing specialists. ${ }^{4,5}$ In addition, residents of Quebec have among the highest rates of visits to emergency departments in international comparative studies. ${ }^{6.8}$
Research is needed into the effect that affiliation with a specialist rather than a family physician has on patients' use of the emergency department, as is research into the continuity and comprehensiveness of care provided by the primary physician, regardless of specialty.

Many studies have shown the tendency for people without a regular care provider to use the emergency department more often than people who have a primary physician. ${ }^{1,9,10}$ Greater continuity of care with a primary physician has also been associated with fewer visits to the emergency department, but much of this research is cross-sectional, making causal interpretation difficult. ${ }^{11}$ Furthermore, the distinction between continuity with a family physician versus a specialist primary physician has not been made, although one American study reported that hav-
Competing interests: Jane McCusker, Roxane Borgès Da Silva, Antonio Ciampi and Steven Sanche have received grant funding from Fonds de recherche en Santé du Québec. No other competing interests were declared.

This article has been peer reviewed.

Correspondence to: Dr. Jane McCusker, jane.mccusker@mcgill.ca

CMAJ 2012. DOI:10.1503 /cmaj.111069 
ing a specialist primary physician was associated with increased use of emergency departments. ${ }^{9}$

We sought to determine whether certain factors predicted patients' subsequent use of emergency departments, such as the specialty of their primary physician, the continuity of care with that physician and the comprehensiveness of care provided by that physician. To determine whether certain subgroups of the population may derive greater benefit from a particular type of care, we examined the potentially modifying effects of demographic factors, health status and previous use of health services. We restricted our study to residents of urban areas of Quebec for three reasons: rural residents visit the emergency department for primary care more often than residents of urban areas; ${ }^{12}$ most specialist care is provided in urban areas; and primary care services in rural areas are more likely to be provided by salaried physicians, whose information is not available in the billing database.

\section{Methods}

\section{Design}

We conducted a retrospective, population-based, cohort study, using information from linked provincial administrative databases (enrollee, physician billing and hospital discharge files) covering a three-year period (Apr. 1, 2003, to Mar. 31, 2006). Primary care variables and

Table 1: Characteristics of the excluded sample (i.e., low users) and the analysis cohort during the two-year baseline and one-year follow-up periods (part 1 of 2)

\begin{tabular}{|c|c|c|c|c|c|}
\hline \multirow[b]{2}{*}{ Characteristic } & \multirow{2}{*}{$\begin{array}{l}\text { Patients with fewer } \\
\text { than } 3 \text { visits to a } \\
\text { physician during } \\
\text { baseline, } \% \\
n=55614\end{array}$} & \multicolumn{4}{|c|}{ Analysis cohort } \\
\hline & & $\begin{array}{c}\text { Total, } \% \\
n=311701\end{array}$ & $\begin{array}{c}\text { No primary } \\
\text { physician, \% } \\
n=11959\end{array}$ & $\begin{array}{l}\text { Family physician, \% } \\
\quad n=271990\end{array}$ & $\begin{array}{c}\text { Specialist, } \% \\
n=27752\end{array}$ \\
\hline \multicolumn{6}{|l|}{ Age, yr } \\
\hline $18-34$ & 40.9 & 24.8 & 46.3 & 22.3 & 34.1 \\
\hline $35-64$ & 54.2 & 56.8 & 47.5 & 58.0 & 51.8 \\
\hline $65-74$ & 3.1 & 11.1 & 3.9 & 11.9 & 8.7 \\
\hline$\geq 75$ & 1.8 & 7.3 & 2.4 & 7.8 & 5.5 \\
\hline \multicolumn{6}{|l|}{ Sex } \\
\hline Male & 63.8 & 41.8 & 54.7 & 40.4 & 46.2 \\
\hline Female & 36.2 & 58.2 & 45.3 & 59.6 & 53.8 \\
\hline \multicolumn{6}{|c|}{ Material deprivation percentile } \\
\hline $1-50$ & 53.1 & 55.0 & 57.0 & 54.7 & 56.4 \\
\hline $51-100$ & 44.9 & 43.0 & 41.1 & 43.3 & 41.5 \\
\hline Missing & 2.0 & 2.1 & 1.9 & 2.1 & 2.1 \\
\hline \multicolumn{6}{|c|}{ Area of residence } \\
\hline Urban & 44.7 & 38.8 & 31.1 & 40.3 & 31.1 \\
\hline Metropolitan & 55.3 & 61.2 & 68.9 & 59.7 & 68.9 \\
\hline \multicolumn{6}{|c|}{$\begin{array}{l}\text { Health status, comorbidity score } \\
\text { percentile }\end{array}$} \\
\hline Low, 0-60 & 76.0 & 56.5 & 59.9 & 55.4 & 63.8 \\
\hline Medium, $61-80$ & 21.5 & 24.4 & 30.9 & 23.9 & 24.8 \\
\hline High, 81-100 & 2.6 & 19.1 & 9.2 & 20.7 & 11.4 \\
\hline \multicolumn{6}{|c|}{$\begin{array}{l}\text { Visits to physicians during baseline, } \\
\text { no. }\end{array}$} \\
\hline $0-2$ & 100.0 & - & - & - & - \\
\hline $3-8$ & - & 48.0 & 91.7 & 43.1 & 64.9 \\
\hline $9-24$ & - & 43.0 & 7.5 & 46.9 & 30.6 \\
\hline$\geq 25$ & - & 9.0 & 0.8 & 10.1 & 4.6 \\
\hline \multicolumn{6}{|c|}{$\begin{array}{l}\text { Time spent in hospital during } \\
\text { baseline, d }\end{array}$} \\
\hline 0 & 97.4 & 85.9 & 93.3 & 86.3 & 79.0 \\
\hline $1-3$ & 1.1 & 4.5 & 2.5 & 4.2 & 7.6 \\
\hline
\end{tabular}


covariables were measured during an initial baseline period (the first two years of the study); visits to emergency departments were measured during the last year of the study (the follow-up period). We selected a stratified random sample of adult enrollees who had at least one claim for health care during the baseline period, with 100000 participants from each of four strata defined by age ( $<65$ years and $\geq 65$ years) and area of residence (metropolitan areas [ $\geq 500000$ residents] and urban areas $[<500000$ residents, but within $30 \mathrm{~min}$ of a secondary or tertiary hospital]). ${ }^{13}$ Exclusion criteria included patient death before Apr. 1, 2005, receiving long-term care at any time during the study or living outside of Quebec at any time during the study. A total of 367315 peo- ple were eligible for inclusion in the final sample. The analysis cohort $(n=311701)$ comprised those participants who had made three or more visits to a physician during the baseline period (Table 1).

The study protocol (Appendix 1, available at www.cmaj.ca/lookup/suppl/doi:10.1503/cmaj .111069/-/DC1.) was approved by the Commission d'accès à l'information and the Research Ethics Committee of St. Mary's Hospital.

\section{Measures}

\section{Primary outcome}

Our primary outcome was the number of visits to an emergency department during the 12 -

Table 1: Characteristics of the excluded sample (i.e., low users) and the analysis cohort during the two-year baseline and one-year follow-up periods (part 2 of 2)

\begin{tabular}{|c|c|c|c|c|c|}
\hline \multirow[b]{2}{*}{ Characteristic } & \multirow{2}{*}{$\begin{array}{l}\text { Patients with fewer } \\
\text { than } 3 \text { visits to } \\
\text { physicians during } \\
\text { baseline, } \% \\
n=55614\end{array}$} & \multicolumn{4}{|c|}{ Analysis cohort } \\
\hline & & $\begin{array}{c}\text { Total, } \% \\
n=311701\end{array}$ & $\begin{array}{c}\text { No primary } \\
\text { physician, \% } \\
n=11959\end{array}$ & $\begin{array}{l}\text { Family physician, \% } \\
n=271990\end{array}$ & $\begin{array}{c}\text { Specialist, \% } \\
n=27752\end{array}$ \\
\hline \multicolumn{6}{|c|}{ Time spent in hospital (continued) } \\
\hline$\geq 4$ & 1.5 & 9.6 & 4.3 & 9.5 & 13.4 \\
\hline \multicolumn{6}{|c|}{$\begin{array}{l}\text { Visits to emergency department } \\
\text { during baseline, no. }\end{array}$} \\
\hline 0 & 70.1 & 61.7 & 63.6 & 61.7 & 60.6 \\
\hline 1 & 20.0 & 20.1 & 20.5 & 20.1 & 20.6 \\
\hline 2 & 5.8 & 8.7 & 8.7 & 8.6 & 9.1 \\
\hline$\geq 3$ & 4.1 & 9.5 & 7.3 & 9.6 & 9.8 \\
\hline \multicolumn{6}{|c|}{ Affiliation with a primary physician } \\
\hline Low user & 100.0 & - & - & - & - \\
\hline None & - & 5.5 & 100.0 & - & - \\
\hline Family physician & - & 84.1 & - & 100.0 & - \\
\hline Specialist & - & 10.3 & - & - & 100.0 \\
\hline \multicolumn{6}{|c|}{$\begin{array}{l}\text { Usual provider continuity index with } \\
\text { a primary physician* }\end{array}$} \\
\hline Low $(<0.40)$ & - & 32.5 & - & 34.7 & 15.2 \\
\hline Medium $(0.40-0.79)$ & - & 51.1 & - & 49.5 & 64.1 \\
\hline High $(0.80-1.0)$ & - & 16.4 & - & 15.8 & 20.7 \\
\hline \multicolumn{6}{|c|}{ Complete annual examinations, no. } \\
\hline 0 & - & 65.8 & - & 59.3 & - \\
\hline 1 & - & 25.0 & - & 29.8 & - \\
\hline$\geq 2$ & - & 9.2 & - & 11.0 & - \\
\hline \multicolumn{6}{|c|}{$\begin{array}{l}\text { Visits to emergency department } \\
\text { during follow-up, no. }\end{array}$} \\
\hline 0 & 82.5 & 76.2 & 78.0 & 75.9 & 77.4 \\
\hline 1 & 12.1 & 15.0 & 14.3 & 15.1 & 14.4 \\
\hline 2 & 3.4 & 4.9 & 4.7 & 5.0 & 4.6 \\
\hline$\geq 3$ & 1.9 & 3.9 & 3.0 & 4.0 & 3.6 \\
\hline
\end{tabular}


month follow-up. A visit was defined as one or more billings for an emergency department on up to two consecutive days. ${ }^{14}$

\section{Independent variables}

We determined affiliation with a primary physician using an algorithm adapted from a previous study. ${ }^{15}$ We coded a family physician as being the primary physician if there had been at least two visits to the same physician or at least one visit during which an annual physical examination was completed. If more than one family physician met these criteria, we considered the primary physician to be the family physician who was visited most often. In the case of a tie, we considered the primary physician to be the physician who had completed the most annual physical examinations. If no annual examination had been completed, we considered the primary physician to be the physician visited most recently.

We coded participants who did not have a primary family physician as having a specialist as their primary physician if there had been at least two visits to the same specialist. All specialties were considered, with the exceptions of anatomic pathology, anesthesiology, medical microbiology and infectious disease, medical biochemistry, neurosurgery, neuropsychiatry, diagnostic radiology, radiation-oncology, nuclear medicine, medical genetics and community health. We considered the specialist who was visited the most often to be the primary

\begin{tabular}{|c|c|}
\hline Interaction & $\operatorname{IRR}(95 \% \mathrm{Cl})$ \\
\hline \multicolumn{2}{|l|}{ Overall } \\
\hline \multicolumn{2}{|c|}{ Affiliation with a primary physician } \\
\hline None v. family physician & $1.12(1.07-1.17)$ \\
\hline None v. specialist & $1.08(1.03-1.14)$ \\
\hline Specialist v. family physician & $1.03(1.00-1.06)$ \\
\hline \multicolumn{2}{|c|}{ Continuity of care with a family physiciant } \\
\hline Low v. high & $1.00(0.97-1.03)$ \\
\hline Medium v. high & $1.00(0.97-1.02)$ \\
\hline \multicolumn{2}{|c|}{ Complete annual examinations with a family physician, no. } \\
\hline 0 v. 2 & $1.23(1.19-1.27)$ \\
\hline 1 v. 2 & $1.10(1.07-1.14)$ \\
\hline \multicolumn{2}{|c|}{ Continuity of care with a specialist $\dagger$} \\
\hline Low v. high & $1.17(1.07-1.28)$ \\
\hline Medium v. high & $1.10(1.01-1.18)$ \\
\hline \multicolumn{2}{|l|}{ Age, 18-64 yr } \\
\hline \multicolumn{2}{|c|}{ Affiliation with a primary physician } \\
\hline None v. family physician & $1.11(1.05-1.16)$ \\
\hline None v. specialist & $1.10(1.04-1.17)$ \\
\hline Specialist v. family physician & $1.00(0.97-1.04)$ \\
\hline \multicolumn{2}{|l|}{ Age, $\geq 65$ yr } \\
\hline \multicolumn{2}{|c|}{ Affiliation with a primary physician } \\
\hline None v. family physician & $1.26(1.18-1.34)$ \\
\hline None v. specialist & $1.11(1.04-1.19)$ \\
\hline Specialist v. family physician & $1.13(1.09-1.17)$ \\
\hline \multicolumn{2}{|c|}{ Visits to physicians during baseline, 3-24 } \\
\hline \multicolumn{2}{|c|}{ Continuity of care with a family physiciant } \\
\hline Low v. high & $0.98(0.95-1.01)$ \\
\hline Medium v. high & $0.99(0.96-1.02)$ \\
\hline \multicolumn{2}{|c|}{ Visits to physicians during baseline, $\geq 25$} \\
\hline \multicolumn{2}{|c|}{ Continuity of care with a family physiciant } \\
\hline Low v. high & $1.17(1.07-1.28)$ \\
\hline
\end{tabular}


physician. In the case of ties, participants were considered to have no affiliation with a primary physician.

We measured continuity of care using the Usual Provider Continuity Index as the proportion of ambulatory visits to the primary physician. ${ }^{16} \mathrm{We}$ determined the comprehensiveness of care provided by family physicians using the number of complete annual physical examinations (indicated by the billing code). ${ }^{17}$ No corresponding measure was available for determining the comprehensiveness of care provided by other specialists.

\section{Covariables}

Our covariables included determinants of emergency department use identified in the literature that could confound or modify the associations between the primary care and outcome variables. ${ }^{1,18-21}$ Sociodemographic measures at the midpoint of the baseline period were age, sex, percentile of material deprivation ${ }^{22}$ (a composite measure determined using census data for area of residence that incorporates education, employment and income) and area of residence. Area of residence was coded as metropolitan or urban using the patient's postal code. ${ }^{13}$ To minimize confounding by comorbidity, we developed a comorbidity (multimorbidity) confounder score (see Appendix 2, available at www.cmaj.ca/lookup/suppl/doi:10 $.1503 / \mathrm{cmaj} .111069 /-/ D C 1)$. We used number of visits to physicians, total number of days spent in hospital and total number of visits to emergency

Table 2: Primary care variables predicting use of emergency department, overall and by patient baseline characteristics* (part 2 of 2)

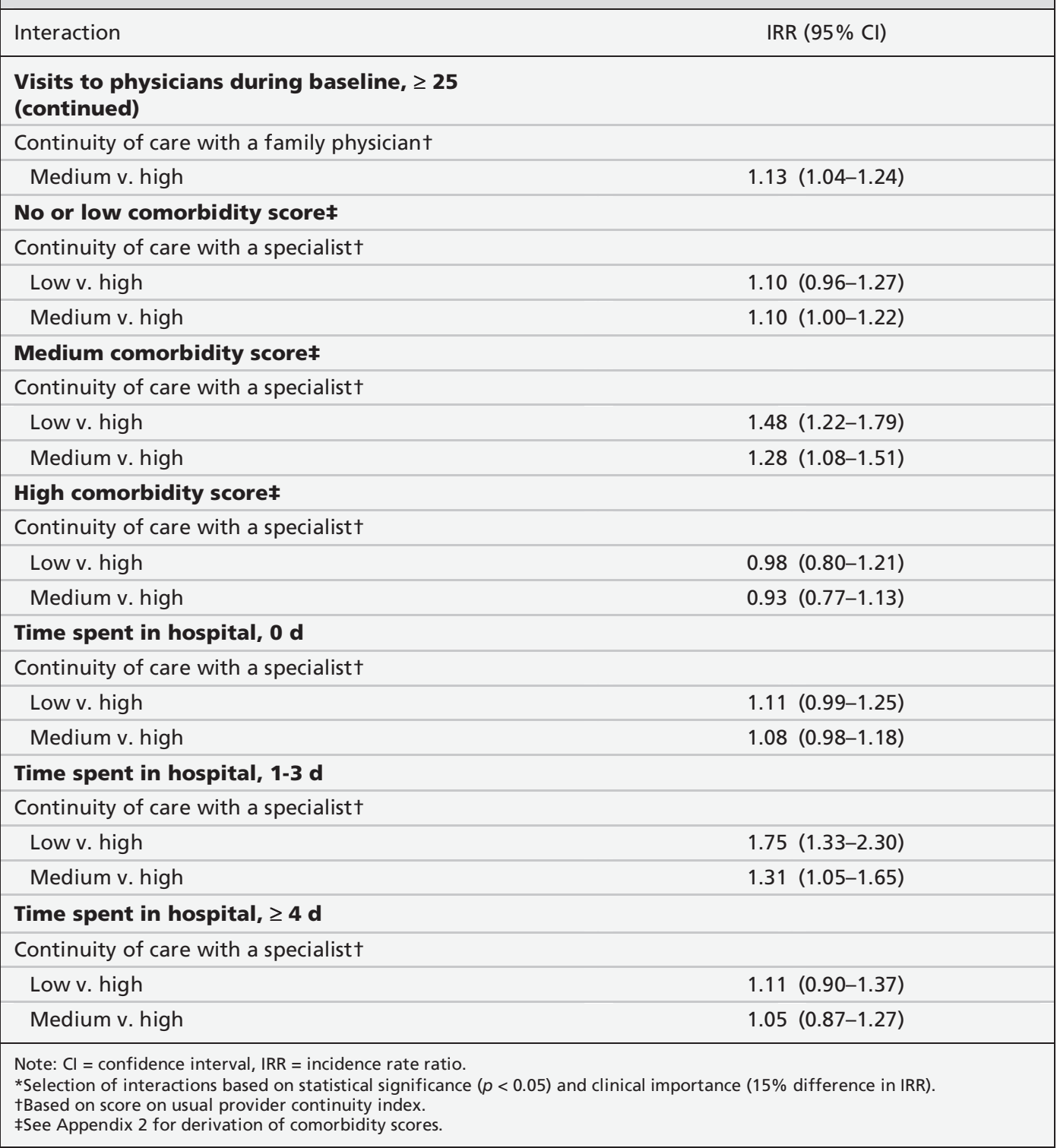


departments as measures of health service use during baseline.

\section{Statistical analysis}

Internal inconsistencies (e.g., unreported deaths, services incorrectly reported as having been provided after a patient's date of death) were corrected in the database. We were missing values only for area of residence (less than $5 \%$ of the sample), in which case we used a similar measure $^{23}$ to conditionally impute a value. ${ }^{24}$

We used negative binomial regression to study the associations of the primary care vari- ables with the number of visits to the emergency department during follow-up, computing incidence rate ratios (IRRs) and their 95\% confidence intervals (CIs). ${ }^{19}$ The effect of continuity of care on the number of visits was estimated for each of the two groups of affiliation (family physician and specialist), whereas the effect of comprehensiveness of care was only estimated for patients affiliated with a family physician. We adjusted all effects for baseline covariables.

We initially input all count or continuous variables as uncategorized in the models. We verified the linearity of the modelled relations by inspect-

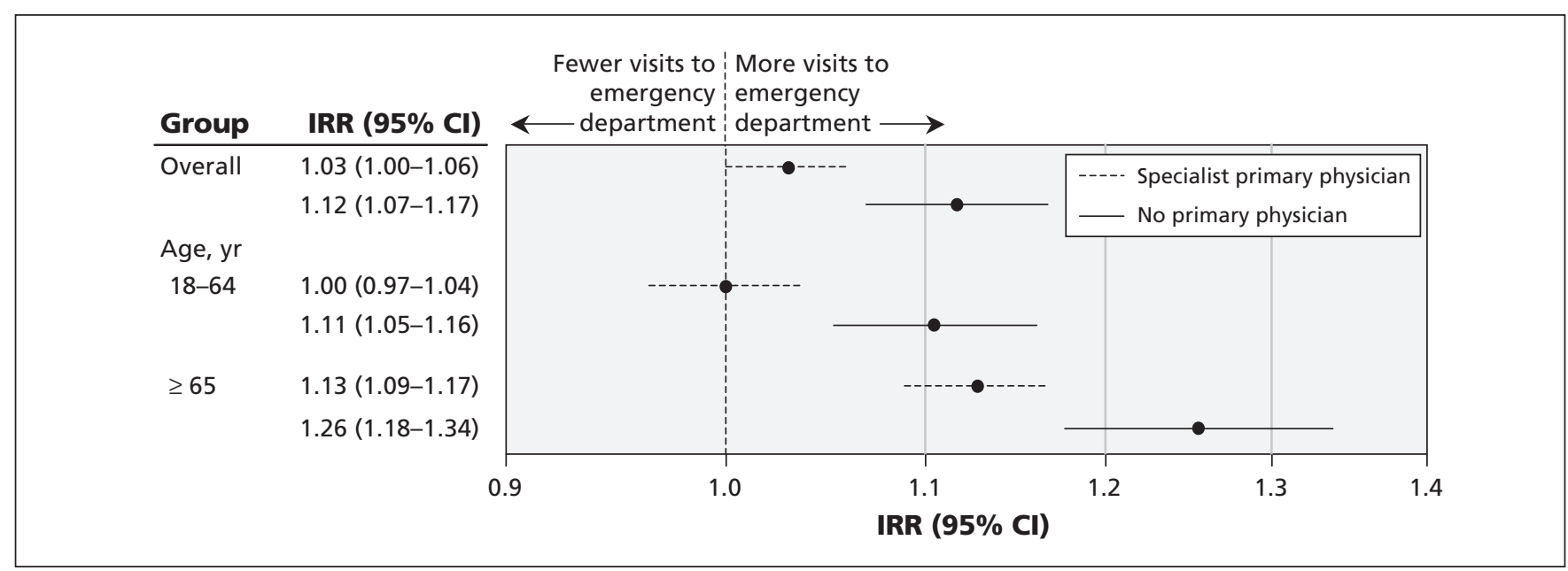

Figure 1: Incidence rate ratios for use of the emergency department by affiliation with a primary physician (overall and stratified by age) among 311701 patients who made three or more physician visits during the baseline period. Models adjusted for age, sex, material deprivation, area of residence, number of physician visits and time spent in hospital during baseline. Patients with a specialist or no primary physician are compared, with patients with a family physician acting as the reference group. $\mathrm{Cl}=\mathrm{Confidence}$ interval, IRR $=$ incidence rate ratio.

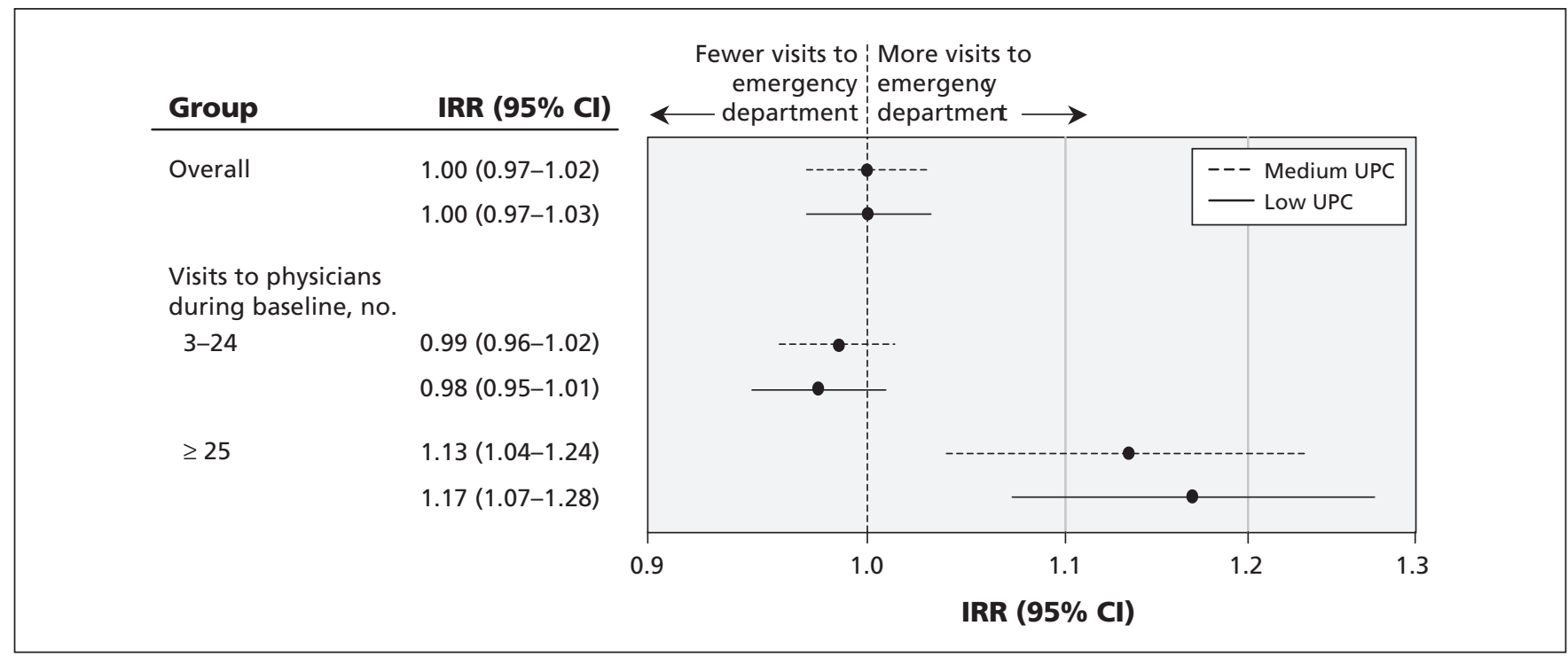

Figure 2: Incidence rate ratios for use of the emergency department by continuity of care by a family physician (overall and stratified by number of doctor visits) among 271990 patients who made three or more visits to physicians during the baseline period, and who have a family physician. Models adjusted for age, sex, material depreivation, area of residence, number of physician visits and time spent in hospital during baseline. Patient with low or medium scores on the Usual Provider Care index are compared, with high scores acting as the reference group. $\mathrm{Cl}=$ confidence interval, IRR = incidence rate ratios, UPC = usual provider continuity. 
ing residual graphs and testing the significance of quadratic terms. When there was departure from linearity, we categorized the count or continuous variables, either using conventional categories or quintiles, with the highest quintile divided in two to capture the effect of the higher end of the scales. We aggregated categories with similar IRRs for parsimony. We explored interactions between the primary care variables and the covariables by adding interaction terms to the main model, using stratified analyses to identify clinically important interactions from among those that were statistically significant.

In the multivariable analyses, we defined a clinically important interaction as a difference of at least $15 \%$ in the IRRs of either two levels of a categorical variable or a change of one standard deviation unit for a continuous variable.

\section{Results}

Characteristics of the excluded sample (i.e., low users) and of the analysis cohort are shown in Table 1. The low users (less than three visits to a physician during baseline) comprised more men and had lower comorbidity scores than the analysis cohort.

We identified several clinically important differences. The group of patients who made fewer than three visits to a physician during baseline

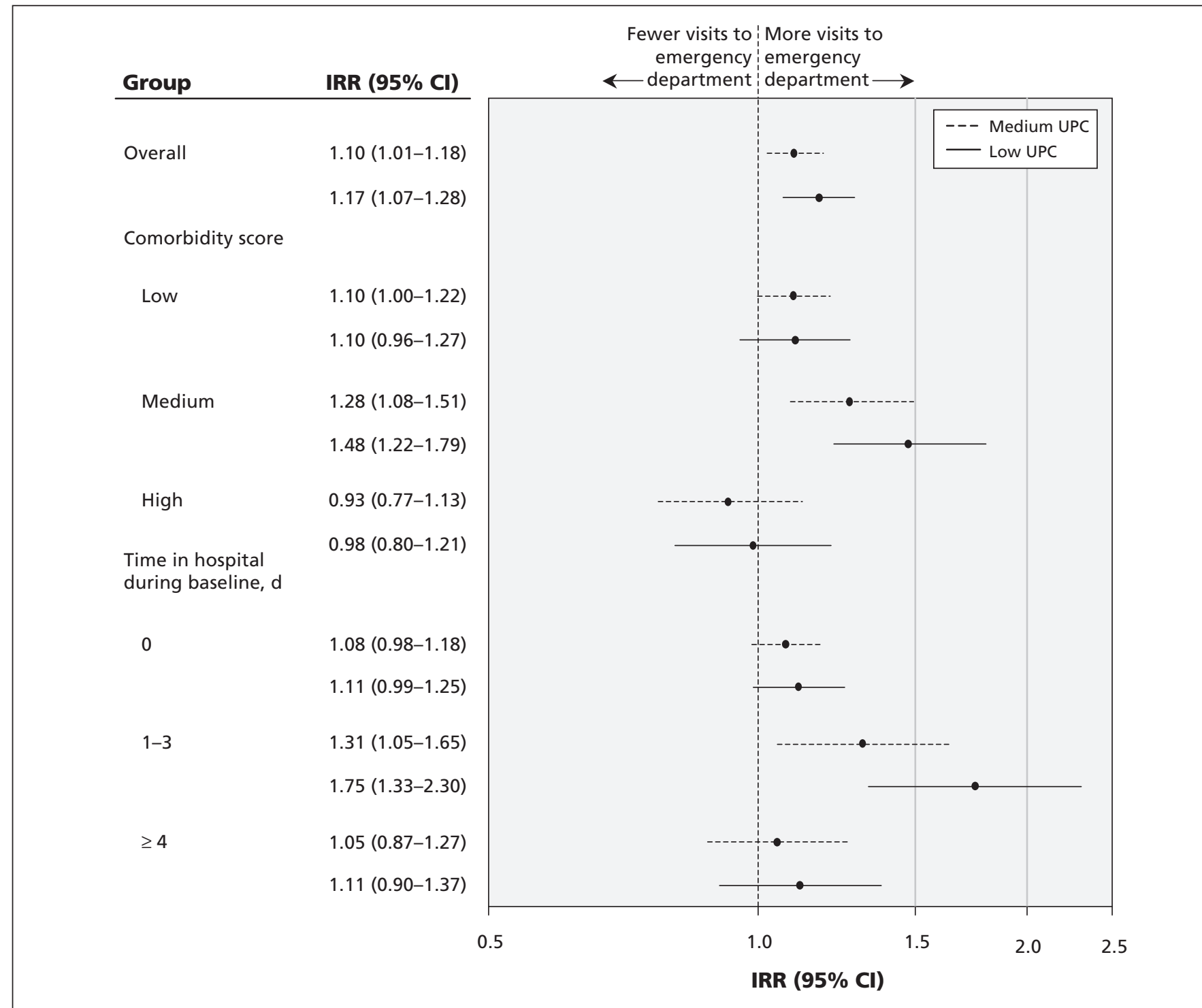

Figure 3: Incidence rate ratios for use of the emergency department by continuity of care by a specialist (overall and stratified by comorbidity score and number of days spent in hospital) among 27752 patients who made three or more visits to physicians during the baseline period and who have a specialist as their primary physician. Models adjusted for age, sex, material deprivation, area of residence, number of physician visits and time spent in hospital during baseline. Patients with low or medium scores on the Usual Provider Care index are compared, with patients with high scores acting as the reference group. $\mathrm{Cl}=\mathrm{confidence}$ interval, IRR $=$ incidence rate ratio, UPC = usual provider continuity. 
comprised more men and had lower comorbidity scores than the analysis cohort. In the analysis cohort, more than four-fifths of the patients were affiliated with a family physician, and 1 in 10 were affiliated with a specialist. In addition, patients with no primary physician were younger, were more likely to be men and had fewer ambulatory visits and admissions to hospital than patients with a primary physician. Finally, patients affiliated with a family physician made more ambulatory visits and had lower continuity of care than patients affiliated with a specialist.

Table 2 shows the multivariable IRRs for the four variables for primary care. In the model without interactions, affiliation with either a family physician or a specialist predicted lower use of the emergency department compared with no affiliation. Among patients with a family physician, continuity of care did not predict use of the emergency department; among patients affiliated with a specialist, lower continuity of care predicted higher use of the emergency department. Among patients with a family physician, greater comprehensiveness of care predicted lower use of the emergency department.

Clinically important interactions are shown in Table 2 and in Figures 1-3. Among patients aged 65 years or more, the beneficial effects of having a family physician were evident in comparison with being affiliated with a specialist or having no primary physician (Figure 1). Although there was no overall effect of continuity of care, among patients who made 25 or more visits to their physician during the baseline period, there was a beneficial effect of greater continuity on subsequent use of the emergency department (Figure 2). The protective effect of greater continuity of care with a specialist appears to be limited to patients with medium comorbidity scores and stays in hospital lasting one to three days (Figure 3). No clinically important interactions were found between the comprehensive of care provided by family physicians and the study covariables (data not shown).

\section{Interpretation}

Our results suggest differences among subgroups in terms of the benefits of being affiliated with a family physician and greater continuity of care with that physician, even after adjustment for demographic characteristics, multiple comorbidities and use of health services.

The benefits of affiliation with a family physician were more apparent among those patients aged 65 years and older. The benefits of greater continuity of care with a family physician were limited to those patients who made frequent visits to doctors.
Our study extends previous research on the benefits of affiliation with a family physician by showing the greater importance of such an affiliation among seniors (i.e., patients aged 65 years and older). ${ }^{25,26}$ Seniors have more complex needs related to cognitive and physical deficits, which can benefit from more coordinated care. ${ }^{27}$ The overall failure of continuity of care with a family physician to predict subsequent use of the emergency department that we saw in this study can be partially explained by the study's design (cohort v. cross-sectional), which avoided a reverse causality bias in which use of the emergency department would lead to reduced continuity. ${ }^{11}$ However, we did see a beneficial effect of greater continuity of care among patients who visit the doctor more frequently (i.e., more than once per month). The need for the coordinating role of a family physician is accentuated among more frequent users of physician services. ${ }^{28}$

In addition, we found potential benefits of more comprehensive care from a family physician, as measured by the number of complete annual physical examinations. However, these results should be interpreted cautiously; they may be caused by self-selection of people who are very health-conscious. Nevertheless, a recent systematic review concluded that a complete annual physical examination improves the delivery of preventive services and may reduce worry in patients. ${ }^{29}$

Our study adds to the information on care provided by specialists in the role of primary physician. The use of specialists in Canada is lower than in the United States and is related more to need than to the ability to pay. ${ }^{30} \mathrm{Al}$ though people who have a specialist as their primary physician use the emergency department at rates similar to people who have a family physician, our results suggest that greater continuity of care with a specialist can be beneficial for patients with medium comorbidity scores and short stays in hospital (i.e., $<3$ days). For healthier people, the need for coordination is less important; for sicker patients, specialists may have difficulty coordinating care because of a lack of expertise outside their traditional scope of practice. In addition, specialists may be less likely than family physicians to provide preventive care, such as vaccinations against influenza. ${ }^{31}$

\section{Limitations}

Our study's limitations are primarily related to our use of administrative databases. For example, there may be residual confounding by unmeasured or inaccurately measured variables. Unmeasured covariables or selection bias may account for differences in the effect of affiliation 
with a specialist rather than a family physician or the effect of continuity of care with a specialist versus a family physician.

We were only able to analyze billed services. Although these services account for more than $98 \%$ of physician services in Quebec, ${ }^{32}$ our results cannot be generalized to the small population whose usual care provider is a salaried physician.

Although we did base our measure of affiliation on a validated algorithm, it may not identify the doctor who patients consider to be their primary physician. In addition, preventive care by family physicians or specialists could not be measured directly. Although statistically significant, many of the effects of primary care we saw were small and may not be clinically important.

Finally, our research was done in the context of health care in Quebec and may not be generalizable to other provinces or countries. ${ }^{4}$

\section{Conclusion}

Our results suggest that certain populations, particularly the elderly, the very sick and patients with chronic disease, derive the most benefit from being affiliated with a family physician as their primary physician. Any financial incentives for family physicians to register more patients would be best aimed at these vulnerable populations, such as has been done in Quebec since $2002 .{ }^{33}$ For younger, healthier populations, the benefits of a family physician are less clear.

Future research should evaluate the effects of emerging models of primary care, financial incentives and other policies designed to increase affiliations with family physicians on use of the emergency department.

\section{References}

1. McCusker J, Karp I, Cardin S, et al. Determinants of emergency department visits by older adults: a systematic review. Acad Emerg Med 2003;10:1362-70.

2. Petersen LA, Burstin HR, O'Neil AC, et al. Nonurgent emergency department visits: the effect of having a regular doctor. Med Care 1998;36:1249-55.

3. Hutchison B, Levesque JF, Strumpf E, et al. Primary health care in Canada: systems in motion. Milbank Q 2011;89:256-88.

4. Dunlop S, Coyte PC, McIsaac W. Socio-economic status and the utilisation of physicians' services: results from the Canadian National Population Health Survey. Soc Sci Med 2000;51:123-33.

5. Sibley LM, Weiner JP. An evaluation of access to health care services along the rural-urban continuum in Canada. BMC Health Serv Res 2011;11:20.

6. Schoen C, Osborn R, Huynh PT, et al. Taking the pulse of health care systems: experiences of patients with health problems in six countries. Health Aff 2005; [Suppl Web exclusives ]:509-25.

7. Schoen C, Osborn R, Doty MM, et al. Toward higherperformance health systems: adults' health care experiences in seven countries. Health Aff (Millwood) 2007;26:W717-34 .

8. Roberge D, Pineault R, Larouche D, et al. The continuing saga of emergency room overcrowding: are we aiming at the right target? Healthc Policy 2010;5:27-39.

9. Rosenblatt RA, Wright GE, Baldwin LM, et al. The effect of the doctor-patient relationship on emergency department use among the elderly. Am J Public Health 2000;90:97-102.

10. Ionescu-Ittu R, McCusker J, Ciampi A, et al. Continuity of primary care and emergency department utilization among elderly people. CMAJ 2007;177:1362-8.
11. van Walraven $\mathrm{C}$, Oakes $\mathrm{N}$, Jennings $\mathrm{A}$, et al. The association between continuity of care and outcomes: a systematic and critical review. J Eval Clin Pract 2010;16:947-56.

12. Haggerty JL, Roberge D, Pineault R, et al. Features of primary healthcare clinics associated with patients' utilization of emergency rooms: urban-rural differences. Healthc Policy 2007;3:72-85.

13. Gauthier J, Haggerty J, Lamarche P, et al. Entre adaptabilité et fragilité: les conditions d'accès aux services de santé des communautés rurales et éloignées. Montréal (QC): Centre de recherche Hôpital Charles LeMoyne and Quebec (QC): Institut National de Santé public; 2009. Available: https://docs.google .com/viewer? $\mathrm{a}=\mathrm{v} \& \mathrm{q}=$ cache:AHHLuMxCVBYJ:www.inspq.qc.ca /pdf (accessed 2010 Oct. 15).

14. Belzile $\mathrm{E}$, Sanche $\mathrm{S}, \mathrm{McCusker} \mathrm{J}$, et al. A measure of emergency department use based on administrative data. Montréal (QC): St. Mary's Hospital Centre; 2011. Available: www.smhc.ca/en/research /our-research/research-projects/emergency-services/special-reports (accessed 2011 July 15).

15. McCusker J, Verdon J, Tousignant P, et al. Rapid emergency department intervention for elders reduces risk of functional decline: results of a multi-center randomized trial. J Am Geriatr Soc 2001;49:1272-81.

16. Breslau N, Reeb KG. Continuity of care in a university-based practice. J Med Educ 1975;50:965-9.

17. Borgès Da Silva R, Contandriopoulos A-P, Pineault R, et al. A global approach to evaluation of health services utilization: concepts and measures. Health Policy 2011;6:e106-17.

18. Chan BTB, Ovens HJ. Frequent users of emergency departments: Do they also use family physicians' services? Can Fam Physician 2002;48:1654-60.

19. Stata Corp LP. Stata release 10: user's guide. College Station (TX): Stata Press LP; 2007

20. Brown EM, Goel V. Factors related to emergency department use: results from the Ontario Health Survey 1990. Ann Emerg Med 1994;24:1083-91.

21. Reid R, Evans R, Barer M, et al. Conspicuous consumption: characterizing high users of physician services in one Canadian province. J Health Serv Res Policy 2003;8:215-24.

22. Pampalon R, Raymond G. A deprivation index for health and welfare planning in Quebec. Chronic Dis Can 2000;21:104-13.

23. McNiven C, Puderer H, Janes D. Census Metropolitan Area and Census Agglomeration Influence Zones (MIZ): a description of the methodology. Ottawa (ON): Statistics Canada; 2000. Available: www.statcan.gc.ca/bsolc/olc-cel/olc-cel?lang=eng\&catno =92F0138M2000002 (accessed 2011 Jan. 15).

24. Arnold BC, Castillo E, Sarabia JM. Conditionally specified distributions: an introduction (with comments and a rejoinder by the authors). Stat Sci 2001;16:249-74.

25. McCusker J, Roberge D, Lévesque J-F, et al. Emergency department visits and primary care among adults with chronic conditions. Med Care 2010;48:972-80.

26. McIsaac WJ, Fuller-Thomson E, Talbot Y. Does having regular care by a family physician improve preventive care? Can Fam Physician 2001;47:70-6.

27. McCusker J, Verdon J. Do geriatric interventions reduce emergency department visits? A systematic review. J Gerontol A Biol Sci Med Sci 2006;61:53-62.

28. Menec VH, Sirski M, Attawar D. Does continuity of care matter in a universally insured population? Health Serv Res 2005;40: 389-400.

29. Boulware LE, Marinopoulos S, Phillips KA, et al. Systematic review: the value of the periodic health evaluation. Ann Intern Med 2007;146:289-300.

30. Kaplan MS, Huguet N, Feeny D, et al. Visits to family physicians or specialists by elderly persons in Canada and the United States: an exploratory comparative study. Int J Health Serv 2010;40:139-49.

31. Rosenblatt RA. The generalist role of specialty physicians: Is there a hidden system of primary care? JAMA 1998;279:1364-70

32. Régie de l'assurance maladie du Québec. Nombre et coût des services médicaux selon le type de service, le mode de rémunération et la région sociosanitaire du dispensateur, Québec. Québec (QC): Stat RAMQ; 2004.

33. Levesque J-F, Roberge D, Pineault R. La première ligne de soins: un témoin distant des réformes institutionelles et hospitalières au Québec? In: Morin G, editor. Le système sociosanitaire au Québec: gouvernance, régulation et participation. Montréal (QC): Chenelière Éducation; 2007. p. 63-78.

Affiliations: From the Department of Epidemiology, Biostatistics and Occupational Health, McGill University, and St. Mary's Research Centre, (McCusker, Ciampi); the Institut national de santé publique du Québec, and Department of 
Epidemiology, Biostatistics and Occupational Health (Tousignant), McGill University; the Department of Epidemiology, Biostatistics and Occupational Health, (Borgès Da Silva) McGill University; the Centre de recherche du Centre hospitalier de l'Université de Montréal et Institut national de santé publique du Québec (Lévesque); the Department of Emergency Medicine Services (Vadeboncoeur), Montreal Institute of Cardiology; and St. Mary's Research Centre (Sanche), Montréal, Que.

Contributors: Jane McCusker obtained funding, led the study, and drafted the paper; Pierre Tousignant helped obtain funding, and provided input into the study's design and the analysis of the data; Roxane Borgès Da Silva provided input into the study's design and the analysis of the data, and assisted with the literature review; Antonio Ciampi helped obtain funding, provided input into the study's design and was responsible for the statistical analyses; Jean-Frédéric Lévesque and Alain Vadeboncoeur helped obtain funding and provided input into the study's design; Steven Sanche conducted the statistical analyses. All of the authors revised the article critically for important intellectual content and approved the final version submitted for publication.

Funding: This work was financed by a grant from Fonds de la recherche en santé du Québec. Roxane Borgès Da Silva was funded by the Canadian Institutes of Health Research and Réseau de recherche en santé des populations du Québec Strategic Training Program in Transdisciplinary Research on Public and Population Health Interventions: Promotion, Prevention and Public Policy (4P). This work was presented in part at the Canadian Association for Health Services and Policy Research (CAHSPR) Annual Conference, Halifax, NS, May 10-13, 2011. 